\title{
Sawtooth-Control Mechanism using Toroidally Propagating Ion-Cyclotron-Resonance Waves in Tokamaks
}

\author{
J. P. Graves, ${ }^{1}$ I. Chapman, ${ }^{2}$ S. Coda, ${ }^{1}$ L.-G. Eriksson, ${ }^{3}$ and T. Johnson ${ }^{4}$ \\ JET-EFDA, Culham Science Centre, OX14 3DB, Abingdon, United Kingdom \\ ${ }^{1}$ École Polytechnique Fédérale de Lausanne (EPFL), Centre de Recherches en Physique des Plasmas, \\ Association EURATOM-Confédération Suisse, 1015 Lausanne, Switzerland \\ ${ }^{2}$ UKAEA/Euratom Fusion Association, Culham Science Centre, Abingdon, United Kingdom \\ ${ }^{3}$ Association EURATOM-CEA, CEA/DSM/IRFM, CEA-Cadarache, F-13108 St. Paul lez Durance, France \\ ${ }^{4}$ Euratom-VR Association, EES, KTH, Stockholm, Sweden
}

(Received 31 July 2008; published 12 February 2009)

\begin{abstract}
The sawtooth control mechanism in plasmas employing off-axis toroidally propagating ion cyclotron resonance waves in tokamaks is reinvestigated. The radial drift excursion of energetic passing ions distributed asymmetrically in the velocity parallel to the magnetic field determines stability when the rational $q=1$ surface resides within a narrow region centered about the shifted fundamental cyclotron resonance.
\end{abstract}

DOI: 10.1103/PhysRevLett.102.065005

PACS numbers: 52.55.Fa, 52.50.Qt, 52.55.Tn

Magnetohydrodynamic (MHD) stability of plasmas in the presence of energetic ions is a crucial issue for present and future large tokamak experiments. Such ions include 3.5 MeV fusion alpha particles, and energetic minority ions produced by auxiliary heating methods such as from ion cyclotron resonance frequency (ICRF) waves. Ions trapped outside the region of highest magnetic field strength have been shown [1] to stabilize a key core instability known as the sawtooth, located within the $q=1$ rational surface, thereby lengthening the period between sequential soft-xray relaxations [2]. Without an effective means of shortening the period of sawteeth, coupling can occur with instabilities located at rational surfaces closer to the tokamak edge. Indeed, interaction of long sawteeth and performance degrading neoclassical tearing modes has been observed [3] in the Joint European Torus (JET), while improved plasma confinement is often found to coincide with small regular sawteeth.

Under certain conditions untrapped, or passing, energetic ions can also strongly influence sawteeth. Sawtooth control from energetic ions injected with near tangential unbalanced neutral beams has already been demonstrated analytically, for deeply passing ions [4], numerically and experimentally [5]. The mechanism responsible was found to be due to the contribution on the $n=1$ internal kink mode of passing particles intersecting the $q=1$ rational surface. An important fast ion effect is obtained when the distribution function of passing ions is asymmetrically distributed in the velocity parallel to the magnetic field. By extending the analysis of Ref. [4] to solve for the internal kink mode across all velocity space, including barely passing trajectories, it is shown in the present contribution that the sawtooth control mechanism responsible for localized off-axis toroidally propagating ICRF waves is essentially the same as for unbalanced neutral beam injection (NBI) scenarios. Moreover, the propagating ICRF waves are more effective than unbalanced NBI because the orbit widths of the energetic ions are larger, and the parallel asymmetry of the distribution function is more strongly radially sheared. Simulations using SELFO [6] for the ICRF wave field and distribution function are applied to a key demonstration JET discharge [7] with localized off-axis ion cyclotron current drive (ICCD) counter to the Ohmic current. Analytical and full numerical calculations [8] of the internal kink mode with the simulated JET ICRF distribution function [9] demonstrate ideal instability when the deposition of the resonating ions is very close to the $q=1$ radius $r_{1}$. Such is the sensitivity to the location of deposition, and the magnitude of the effect, that this fast ion mechanism, i.e., non-MHD mechanism, dominates over the previously assumed classical mechanism (e.g., Refs. [9-11]) relating to the change in the magnetic shear due to the fast ions [12], and the resulting effect on MHD stability.

In the absence of an electrostatic potential, the equilibrium distribution of fast particles $F$ depends on the kinetic energy $\mathcal{E}=v^{2} / 2$, the magnetic moment $\mu=v_{\perp}^{2} / B$, the toroidal canonical momentum $\mathcal{P}_{\phi}=R v_{\phi}+Z e \psi_{p} / m_{h}$, and $\sigma= \pm 1$, which depends on the sign of $v_{\|}$. Here, $\psi_{p}$ is related to the minor radius $r$ by $r B_{0} d r \approx q(r) d \psi_{p}$. Let us expand $F$ in orders of the orbit width $\Delta_{r}$, so that $F=$ $F_{0}+F_{1}+\ldots$ A useful alternative to $\mathcal{P}_{\phi}$ is the equivalent constant of motion $\bar{r}=\tau_{b}^{-1} \int_{0}^{\tau_{b}} d \operatorname{tr}(t)$, where $\tau_{b}$ is the bounce time, or transit time, for, respectively, trapped or passing particles. Writing $r(t)=\bar{r}+\Delta_{r}(t)$ we have

$$
\begin{aligned}
F_{0} & =\left.F(\mathcal{E}, \mu, \bar{r})\right|_{\bar{r} \rightarrow r} \\
\text { and } \quad F_{1}(\mathcal{E}, \mu, r) & =-\Delta_{r} G_{0}(\mathcal{E}, \mu, r)
\end{aligned}
$$

with

$$
G_{0}(\mathcal{E}, \mu, r)=\left.G(\mathcal{E}, \mu, \bar{r})\right|_{\bar{r} \rightarrow r} \quad \text { and } \quad G=\frac{\partial F}{\partial \bar{r}} .
$$


The finite orbit expansion allows separation of two types of toroidal current $\left(j_{\phi} \equiv e Z \int d v^{3} v_{\phi} F\right)$ drive effects. Now, defining $F^{ \pm}=F(\sigma)$, and using $v_{\phi} \approx v_{\|}$and $\int d v^{3}=\sum_{\sigma} \pi \int_{0}^{\infty} d \mathcal{E}(2 \mathcal{E}) \int_{0}^{1 / B} d \lambda \frac{B}{\left|v_{\|}\right|}$, yields the expansion of the current $j_{\phi}=j_{\phi_{0}}+j_{\phi 1}$, where

$$
\begin{aligned}
& j_{\phi_{0}}=Z e \pi \int_{0}^{\infty} d \mathcal{E}(2 \mathcal{E}) \int_{0}^{1 / B} d \lambda B p\left(F_{0}^{+}-F_{0}^{-}\right), \\
& j_{\phi_{1}}=-Z e \pi \int_{0}^{\infty} d \mathcal{E}(2 \mathcal{E}) \int_{0}^{1 / B} d \lambda B \Delta_{r}\left(G_{0}^{+}+G_{0}^{-}\right),
\end{aligned}
$$

with $\Delta_{r}=q\left(\left|v_{\|}\right| R-p R_{0}^{2} q \omega_{b}\right) /\left(r \Omega_{c}\right)$, where $p=1$ for passing particles, $p=0$ for trapped particles, $\omega_{b}=$ $2 \pi / \tau_{b}$, and $\Omega_{c}=e Z B_{0} / m$. We note that the current driven entirely by passing ions $j_{\phi_{0}}$ can encompass both Fisch currents [12], and the currents associated with detrapping into preferentially co- or counter-passing ions $[9,13]$. Both require an asymmetric distribution in $v_{\|}$at the lowest order, i.e., in $F_{0}$. The finite orbit corrected current $j_{\phi_{1}}$ is dominated by trapped ions, and is associated with the constraint of canonical momentum conservation and radial gradients in the distribution function.

We now examine the internal kink stability in the presence of general asymmetrically distributed ions. The relevant ordering employed is $\beta \sim O\left(\epsilon^{2}\right)$, where $\beta=$ $2 \mu_{0} P_{h, c} / B_{0}^{2}$ is the ratio of hot, or core, particle pressure and the magnetic pressure, and $\epsilon=r / R_{0}$ is the inverse aspect ratio. The fast ion perturbed distribution function $\delta F=\delta F_{f}+\delta F_{k}$, where [4]

$$
\delta F_{f}=-(Z e / m)\left(\boldsymbol{\xi} \cdot \nabla \psi_{p}\right) \frac{\partial F}{\partial \mathcal{P}_{\phi}}
$$

is the adiabatic (fluid) contribution, $\boldsymbol{\xi}=\hat{\boldsymbol{\xi}} \exp (-i n \phi-$ $i \omega t)$ is the MHD displacement with $\hat{\boldsymbol{\xi}}=$ $\sum_{m} \hat{\boldsymbol{\xi}}_{m} \exp (-i m \theta)$, and the nonadiabatic (kinetic) contribution $\delta F_{k}$ can be approximately written as "bounce time" $\tau_{b}$ periodic function of time [4,14]:

$$
\begin{aligned}
\delta F_{k} & =\sum_{l=-\infty}^{\infty} \delta F_{k}^{(l)} \exp \left[-i\left(\omega+l \omega_{b}+n\langle\dot{\phi}\rangle\right) t\right], \\
\delta F_{k}^{(l)}= & -\frac{\omega-n \omega_{*}}{\omega+n\langle\dot{\phi}\rangle+l \omega_{b}} \frac{\partial F}{\partial \mathcal{E}} \\
& \times\left\langle\left(v_{\|}^{2}+\frac{v_{\perp}^{2}}{2}\right) \boldsymbol{\kappa} \cdot \boldsymbol{\xi}_{\perp} \exp \left[i\left(\omega+l \omega_{b}+n\langle\dot{\phi}\rangle\right) t\right]\right\rangle,
\end{aligned}
$$

where $\omega_{*}=\partial F / \partial \mathcal{P}_{\phi}(\partial F / \partial \mathcal{E})^{-1}, \boldsymbol{\kappa}$ is the magnetic curvature vector and $\langle X\rangle=\frac{1}{\tau_{b}} \int_{0}^{\tau_{b}} d t X$.

In order to relate Eqs. (3) and (4) to a potential energy $\delta W$, we note that $\delta W=-(1 / 2) \int d^{3} x \boldsymbol{\xi}_{\perp}^{*} \cdot \boldsymbol{\delta} \mathcal{F}$, where $\boldsymbol{\delta} \mathcal{F}=\boldsymbol{\delta} \boldsymbol{j} \times \boldsymbol{B}+\boldsymbol{j} \times \boldsymbol{\delta} \boldsymbol{B}-\boldsymbol{\nabla} \cdot \underline{\delta P}$. The fast ion distribution function primarily influences the perturbed pressure tensor $\delta P$. The modification of the fast ions to the current in $\delta \mathcal{F}$ is negligible [14] for the ideal MHD leading order displacement $\hat{\xi}_{r}=\xi_{0} H\left[r_{1}-r\right] \exp (-i \theta)$, where $H$ is the
Heaviside step function. The hot ion contribution is then $\delta W=(m / 2) \int d^{3} x \int d^{3} v \boldsymbol{\kappa} \cdot \boldsymbol{\xi}_{\perp}^{*}\left(v_{\|}^{2}+v_{\perp}^{2} / 2\right) \delta F$.

We retain only the most important kinetic contributions to sawtooth relevant modes, for which it is appropriate to employ $\omega=0$. The most important contribution to Eqs. (4) and (5) is absent of bounce harmonics, i.e., $l=$ 0 . The $l=1$ response $[4,15]$ is small for $\omega=0$. Recalling Eq. (2) for the definition $G_{0}$, we have for the passing ions the leading order (in $\Delta r$ ) kinetic response:

$$
\begin{aligned}
\delta \hat{W}_{k p}= & -\frac{2^{3 / 2}}{\pi^{1 / 2} \epsilon_{1}^{2} R_{0}} \int_{0}^{r_{1}} d r \int_{0}^{1} d y^{2}\left[\frac{2}{\pi}\left(G_{3}+\frac{G_{4}}{2}\right)\right]^{2} \\
& \times\left(\frac{q}{\Omega_{c}}\right)\left(\frac{e T_{\perp}}{m}\right)^{1 / 2}\left(\frac{2 \mu_{0}}{B_{0}^{2}}\right)\left(C^{+}-C^{-}\right)
\end{aligned}
$$

while for trapped ions one obtains the well known trapped ion kinetic response $\delta \hat{W}_{k t}$, defined, e.g., in Eq. (11) of Ref. [16]. In the above

$$
C^{\sigma}=\left(\frac{e T_{\perp}}{m}\right)^{-1 / 2} \frac{(m / 2)(\pi / 2)^{3 / 2}}{\left[y^{2}+\epsilon\left(2-y^{2}\right)\right]^{3}} \int_{0}^{\infty} d \mathcal{E}(2 \mathcal{E})^{2} G_{0}^{\sigma},
$$

and $\delta \hat{W}=\delta W /\left(2 \pi^{2} R_{0} \xi^{2} \epsilon_{1}^{2} B_{0}^{2} / \mu_{0}\right)$, together with

$$
\begin{aligned}
G_{3} & =\frac{\epsilon}{2} \int_{-\pi}^{\pi} d \theta J(\theta)(1-\epsilon \cos \theta)\left[1-y^{2} \sin ^{2}(\theta / 2)\right]^{1 / 2}, \\
G_{4} & =\frac{y^{2}}{4} \int_{-\pi}^{\pi} d \theta J(\theta) \frac{(1-\epsilon \cos \theta)^{2}}{\left[1-y^{2} \sin ^{2}(\theta / 2)\right]^{1 / 2}}
\end{aligned}
$$

where $J(\theta)=\cos \left(\theta q-\pi K\left[\theta / 2, y^{2}\right] / K\left[y^{2}\right]\right), \quad K\left[\phi, y^{2}\right]$, and $K\left[y^{2}\right]$ are, respectively, incomplete and complete elliptic integrals of the first kind, and the pitch angle $y^{2}=$ $2 \lambda B_{0} /\left[1-\lambda B_{0}(1-\epsilon)\right]$, with $\lambda=\mu / \mathcal{E}$.

In order to identify finite orbit effects, we expand Eq. (3) about the flux label $r$. For this purpose we note that $\boldsymbol{\xi} \cdot \boldsymbol{\nabla} \psi=r B_{0} \xi_{r} / q(r)$ and $\partial / \partial \mathcal{P}_{\phi}=\Omega_{c}^{-1}(q(\bar{r}) / \bar{r}) \partial / \partial \bar{r}$, $r / \bar{r}=1+\Delta_{r} / r, q(\bar{r}) / q(r)=1-\Delta_{r} s(r) / r$. Thus

$$
\begin{aligned}
\delta F_{f}= & -\xi_{r}\left[G_{0}+\frac{\Delta_{r}}{r}\left((2-s) G_{0}\right.\right. \\
& \left.\left.-\left.\frac{y^{2}}{2}\left(2-y^{2}\right) \frac{\partial G_{0}}{\partial y^{2}}\right|_{r}-\left.\frac{\partial\left(r G_{0}\right)}{\partial r}\right|_{y^{2}}\right)\right]
\end{aligned}
$$

where $\partial G /\left.\partial r\right|_{\lambda}=\partial G /\left.\partial r\right|_{y^{2}}+\left(2-y^{2}\right) y^{2}(2 r)^{-1} \partial G /\left.\partial y^{2}\right|_{r}$.

The $\delta W$ contribution corresponding to the first term in Eq. (7) yields the usual adiabatic response in the absence of finite orbit effects $\delta \hat{W}_{f 0}$ (see, e.g., Eqs. (19) and (20) of Ref. [16]) and is generally the same order of magnitude as $\delta \hat{W}{ }_{k t}$. The terms in Eq. (7) proportional to $\Delta r$ are written in a convenient form upon integration by parts: $\delta \hat{W}_{f p 1}=$ $\delta \hat{W}_{r_{1}}+\delta \hat{W}_{y^{2}=1}+\delta \hat{W}_{f p 1}($ extra), where 


$$
\begin{aligned}
\delta \hat{W}_{f p 1}(\text { extra })= & \frac{2^{3 / 2}}{\pi^{1 / 2} \epsilon_{1}^{2} R_{0}} \int_{0}^{r_{1}} d r \int_{0}^{1} d y^{2}\left(\frac{q}{\Omega_{c}}\right)\left(\frac{e T_{\perp}}{m}\right)^{1 / 2} \\
& \times\left[\epsilon y^{2}-\left(\frac{y^{2}}{2}+\epsilon \frac{\partial}{\partial \epsilon}+\frac{y^{2}}{2}\left(2-y^{2}\right) \frac{\partial}{\partial y^{2}}\right)\right. \\
& \left.\times\left(\frac{2 G_{1}+G_{2}}{K\left[y^{2}\right]}\right)\right]\left(\frac{2 \mu_{0}}{B_{0}^{2}}\right)\left(C^{+}-C^{-}\right),
\end{aligned}
$$

$G_{1}=\frac{\epsilon}{2} \int_{-\pi}^{\pi} d \theta \cos \theta(1-\epsilon \cos \theta)\left[1-y^{2} \sin ^{2}(\theta / 2)\right]^{1 / 2}$,

$G_{2}=\frac{y^{2}}{4} \int_{-\pi}^{\pi} d \theta \cos \theta(1-\epsilon \cos \theta)^{2}\left[1-y^{2} \sin ^{2}(\theta / 2)\right]^{-1 / 2}$.

Now, $\delta \hat{W}_{f p 1}($ extra) is almost cancelled by the kinetic contribution for passing ions given by Eq. (6). Note that we have exactly $\delta \hat{W}_{f p 1}$ (extra) $+\delta \hat{W}_{k p}=0$ when all the ions are deeply passing [4], for which $y=0$. The term $\delta W_{y^{2}=1}$ is evaluated on the passing side of the passingtrapped boundary $\left(y^{2}=1\right)$, and hence $\delta \hat{W}_{y^{2}=1}=0$ if $F_{0}$ is symmetric in $v_{\|}$for the trapped ions, and continuous across the passing-trapped boundary. The only significant finite orbit correction term remaining is $\delta \hat{W}_{r_{1}}$ evaluated at $r_{1}$ :

$$
\begin{aligned}
\delta \hat{W}_{r_{1}}= & -\left(\frac{2}{\pi}\right)^{1 / 2} \frac{2}{\epsilon_{1}} \int_{0}^{1} d y^{2}\left(\frac{\epsilon_{1} y^{2}}{2}-\frac{2 G_{1}+G_{2}}{K\left[y^{2}\right]}\right)\left(\frac{q}{\Omega_{c}}\right) \\
& \times\left.\left(\frac{e T_{\perp}}{m}\right)^{1 / 2}\left(\frac{2 \mu_{0}}{B_{0}^{2}}\right)\left(C^{+}-C^{-}\right)\right|_{r=r_{1}}
\end{aligned}
$$

and represents the effect of having nonzero parallel energy flux [17] gradient $\int d v^{3} G_{0}\left(v_{\perp}^{2} / 2+v_{\|}^{2}\right) v_{\|}$at $r_{1}$.

The model ICRF distribution function

$$
\begin{aligned}
F= & \left(\frac{m}{2 \pi e T_{\perp}(\bar{r})}\right)^{3 / 2} A(\bar{r})^{1 / 2} n_{c}(\bar{r})[1+\sigma c(\bar{r}, \lambda)] \\
& \times \exp \left[-\frac{m \mathcal{E}}{e T_{\perp}(\bar{r})}\left(\lambda B_{c}+A(\bar{r})\left|1-\lambda B_{c}\right|\right)\right]
\end{aligned}
$$

involves the resonant magnetic field $B_{c} \equiv B_{0}\left(1-\epsilon_{c}\right)$ [18], anisotropy $\left(A=T_{\perp} / T_{\|}\right.$, with temperatures in units of electron volts), and an asymmetry coefficient $c(\bar{r}, \lambda)$ affecting only the odd moments of the distribution function. Thus

$$
\begin{aligned}
C^{+}-C^{-}= & \frac{e T_{\perp}(r)}{D\left(r, y^{2}\right)^{3}}\left\{\frac{\partial}{\partial r}\left(n_{c} A^{1 / 2} c\right)+n_{c} A^{1 / 2} \frac{y^{2}}{2 r}\left(2-y^{2}\right)\right. \\
& \left.\times \frac{\partial c}{\partial y^{2}}-3 \frac{d A}{d r}\left(\frac{n_{c} A^{1 / 2} c\left|2 \epsilon-y^{2}\left(\epsilon-\epsilon_{c}\right)\right|}{D\left(r, y^{2}\right)}\right)\right\}
\end{aligned}
$$

where $\quad D\left(r, y^{2}\right)=y^{2}\left(1-\epsilon_{c}\right)+A\left|2 \epsilon-y^{2}\left(\epsilon-\epsilon_{c}\right)\right|$. Moreover, the lowest order flux averaged current is

$$
\left\langle j_{\phi 0}(r)\right\rangle=\operatorname{Zen}_{c}\left(\frac{2 e T_{\perp} A}{\pi m}\right)^{1 / 2} \int_{0}^{1} d y^{2} \frac{2 \epsilon c\left(r, y^{2}\right)}{D\left(r, y^{2}\right)^{2}} .
$$

It is found that the contribution of standard passing ions to the finite orbit width corrected current $\left\langle j_{\phi 1}(r)\right\rangle$ is negligible compared to $\left\langle j_{\phi 0}(r)\right\rangle$. Hence, $\left\langle j_{\phi 0}(r)\right\rangle$ and $\left\langle j_{\phi 1}(r)\right\rangle$ can be identified with, respectively, the passing and trapped ion currents, before the bulk plasma drag [10] has been subtracted. The asymmetry function $c\left(r, y^{2}\right)$ can be resolved if the passing ion current contribution is known. We let $c=$ $c_{r}(r) c_{y}\left(y^{2}\right)$, and choose $c_{y}$ to be log-normal in $1-y^{2}$ for $0 \leq y \leq 1$, and $c_{y}=0$ for $y \geq 1$, so that the asymmetry in $F_{0}$ vanishes smoothly at, and beyond, the boundary of passing orbits as required.

We now apply these results to JET discharge 58934, a demonstration discharge described in detail in Ref. [9]. The passing ion current after plasma drag has been subtracted, i.e., the net passing ion current, from the SELFO simulation is shown in Fig. 19(a) of Ref. [9]. Using Eq. (12) and $c\left(r, y^{2}\right)$ described above, we are now able to approximate the passing ion current. The gross passing ion current, i.e., before subtraction of plasma drag, is shown in Fig. 1(a). Additional knowledge of the flux averaged fast ion density and pressure profiles and the anisotropy enables full reconstruction of the model distribution function of the on-axis and off-axis ICRF population of JET discharge 58934. Figure 1(b) illustrates the sensitivity of the $\delta W$ contributions with respect to $r_{1} / a$ for an unchanged fast ion distribution function. The dot-dash curve shows the conventional fast ion contribution $\delta \hat{W}_{0}=\delta \hat{W}_{f 0}+\delta \hat{W}_{k t}$, i.e., the contribution without finite orbit effects. Except for the $q$ profile, all plasma and fast ion related profiles remain fixed. It is seen that as $r_{1}$ increases, $\delta \hat{W}_{0}$ increases, and this result is consistent with the dependence of $-\int_{0}^{r_{1}} d r r^{3 / 2} P_{h}^{\prime} / r_{1}^{2}$ on $r_{1}$. The solid line in Fig. 1(b) plots

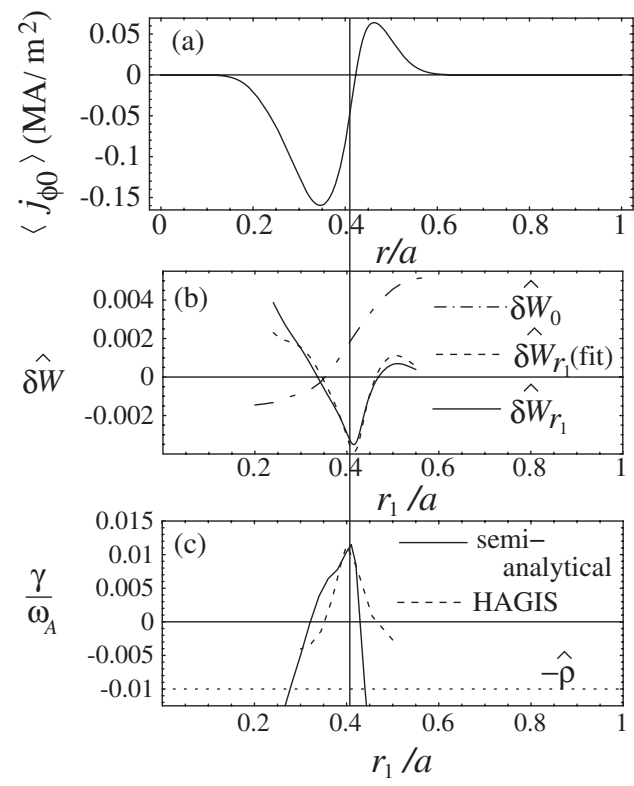

FIG. 1. (a) Using Eqs. (10) and (12) as a fit of the flux averaged passing ion current profile of JET discharge 58934 [10], without bulk plasma drag current. (b) $\delta \hat{W_{0}}$ and $\delta \hat{W_{r 1}}$ according to Eq. (9), and also $\delta \hat{W}_{r 1}$ according to the fit of Eq. (13), plotted as a function of $r_{1} / a$. (c) The fast ion growth rate as a function of $r_{1} / a$, compared with HAGIS simulations. 
the finite orbit correction $\delta \hat{W}_{r_{1}}$, given by Eq. (9), as a function of $r_{1}$. It is seen that there is a deep and narrow minimum in $\delta \hat{W}_{r_{1}}$ close to $r_{1} / a=0.41$, which is close to the measured inversion radius of $r_{\text {inv }} / a=0.34$.

Some simple algebra and numerical integration over pitch angle $y^{2}$ reveals the physics behind the extreme sensitivity of $\delta \hat{W}_{r_{1}}$ to the location of $r_{1}$, since

$$
\delta \hat{W}_{r_{1}} \approx-\left.\frac{2}{\pi \epsilon_{1}} \frac{1}{Z \Omega_{c}}\left(\frac{2 \mu_{0}}{B_{0}^{2}}\right) T_{\perp}^{1 / 2} T_{\|}^{1 / 2} \frac{d\left\langle j_{\phi 0}\right\rangle}{d r}\right|_{r_{1}} .
$$

The dashed line of Fig. 1(b) plots Eq. (13), for the current profile of Fig. 1(a), and it is seen that there is excellent agreement with Eq. (9). It is therefore clear that maximum instability occurs when the largest radial gradient of the passing ion current dipole is at $r_{1}$. The zero in the current dipole, and the shifted resonance position, would then typcially coincide with $r_{1}$. Note that for copropagating waves on the high field side of the device, the current dipole profile reverses sign, and consequently, the corresponding plot of $\delta W_{r_{1}}$ with respect to $r_{1}$ would have the opposite sign to that shown in Fig. 1(b). Hence, for copropagating waves, the shifted resonance location coinciding with $r_{1}$ would be the most stable situation for the internal kink mode. Moreover, the mechanism can also explain sawtoothing experiments where co- or counterICCD is deposited close to $q=1$ on the low field side.

Finally, we compare the above semianalytical results with fully numerical results obtained by feeding the unadulterated SELFO generated fast ion distribution function into the drift kinetic code HAGIS [8], together with an MHD displacement supplied from linear ideal MHD numerical calculations, and evaluating the corresponding fast ion contribution to the potential energy without approximation. The fast ion growth rates $\gamma / \omega_{A}=\left(\pi / s_{1}\right) \delta \hat{W}$, with $\omega_{A}$ the Alfvén frequency, calculated using HAGIS are compared with the net contribution from the semianalytical work contained in this section, i.e., the sum of $\delta \hat{W}_{0}$ together with Eqs. (6), (8), and (9), and are plotted in Fig. 1(c). The narrow peak in the growth rate is clearly recovered in the HAGIS simulations, and it has furthermore been confirmed that it is primarily the passing ions that are responsible for the signature shown. HAGIS also accounts for the finite orbit width of trapped ions, and nonstandard orbits. Away from the location of shifted resonance, this neglect probably accounts for the overly enhanced stability in the analytical calculations. Also shown is the instability threshold $-\hat{\rho}=\rho / r_{1}$ for the resistive-two-fluid internal kink mode [19], with $\rho$ the ion Larmor radius. Neveretheless, due to the dominant effect of the ICRF ions, contributions from the core plasma, or NBI distributions, are unlikely to prevent ideal instability.

In conclusion, a newly discovered mechanism has been proposed that can explain the highly effective nature of sawtooth control using off-axis toroidally propagating ion cyclotron resonance waves. Energetic passing ions influence the internal kink mode when the distribution of ions is asymmetric in $v_{\|}$, a natural feature of toroidally propagating ICRF waves. It is shown that when a counterpropagating wave is deposited sufficiently accurately on the high field side, the fast ion effect is so strong that the internal kink mode is driven ideally unstable, and this in turn is consistent with the observed sawteeth $[7,9]$ that are much shorter in period than those obtained in Ohmic plasmas. This should be contrasted with the classical [10] sawtooth control mechanism relating to the change in the magnetic shear, due to ICCD, which leads to a moderate effect on the threshold of an instability to resistive MHD (e.g., [19]), but with no realistic recourse to ideal instability. Furthermore, unlike the classical sawtooth control mechanism, the fast ion mechanism is independent of the electron drag, which is expected [20] to limit the current drive efficiency of the proposed ICRF system for ITER [21].

We are grateful to Rudi Koslowski for his comments. This work, supported by the Swiss National Science Foundation and by the European Communities under contract of association between EURATOM and Confédération Suisse, was carried out within the framework of the European Fusion Development Agreement. I. C. was supported by the UK Engineering and Physical Sciences Research Council.

[1] D. J. Campbell et al., Phys. Rev. Lett. 60, 2148 (1988); R. B. White et al., ibid. 60, 2038 (1988).

[2] S. von Goeler, W. Stodiek, and N. Sauthoff, Phys. Rev. Lett. 33, 1201 (1974).

[3] O. Sauter et al., Phys. Rev. Lett. 88, 105001 (2002).

[4] J P. Graves, Phys. Rev. Lett. 92, 185003 (2004).

[5] I. Chapman et al., Plasma Phys. Controlled Fusion 50, 045006 (2008).

[6] J. Hedin et al., Nucl. Fusion 42, 527 (2002).

[7] L.-G. Eriksson et al., Phys. Rev. Lett. 92, 235004 (2004).

[8] S. D. Pinches et al., Comput. Phys. Commun. 111, 133 (1998).

[9] L.-G. Eriksson et al., Nucl. Fusion 46, S951 (2006).

[10] V. P. Bhatnagar et al., Nucl. Fusion 34, 1579 (1994).

[11] E. Westerhof et al., Nucl. Fusion 42, 1324 (2002).

[12] N. J. Fisch et al., Rev. Mod. Phys. 59, 175 (1987).

[13] T. Hellsten, J. Carlsson, and L.-G. Eriksson, Phys. Rev. Lett. 74, 3612 (1995).

[14] F. Porcelli et al., Phys. Plasmas 1, 470 (1994).

[15] R. Betti and J. P. Freidberg, Phys. Rev. Lett. 70, 3428 (1993).

[16] J. P. Graves et al., Phys. Plasmas 10, 1034 (2003).

[17] F. L. Hinton and R. D. Hazeltine, Rev. Mod. Phys. 48, 239 (1976).

[18] J. P. Graves et al., Phys. Rev. Lett. 84, 1204 (2000).

[19] F. Porcelli, D. Boucher, and M. N. Rosenbluth, Plasma Phys. Controlled Fusion 38, 2163 (1996).

[20] M. Laxaback and T. Hellsten, Nucl. Fusion 45, 1510 (2005).

[21] ITER Physics Basis et al., Nucl. Fusion 39, 2137 (1999). 